\title{
Intermédialités
}

Histoire et théorie des arts, des lettres et des techniques

Intermediality

History and Theory of the Arts, Literature and Technologies

\section{Naître en paradis}

\section{Jean-Paul Gauthier}

Numéro 1, printemps 2003

Naître

URI : https://id.erudit.org/iderudit/1005445ar

DOI : https://doi.org/10.7202/1005445ar

Aller au sommaire du numéro

Éditeur(s)

Centre de recherche sur l'intermédialité

ISSN

1920-3136 (numérique)

Découvrir la revue

Citer cet article

Gauthier, J.-P. (2003). Naître en paradis. Intermédialités / Intermediality, (1), 65-74. https://doi.org/10.7202/1005445ar
Résumé de l'article

Si l'on a pris l'habitude de lire un de nos mythes fondateurs, celui d'Adam et Ève, comme témoignant du sort défavorable attaché à l'être humain, c'est que l'on a épousé le point de vue de Dieu pour lequel le bien et le mal s'opposent radicalement. Ce texte prend le contre-pied d'une telle lecture en épousant le choix de nos premiers parents en faveur de la contingence, ce qui, d'une part nous permet de réviser le concept de liberté, ce qui nous engage, d'autre part, à éprouver la relativité du bien et du mal, lesquels n'ont de cesse en chaque geste de s'entremettre. 


\title{
Naître en paradis
}

\author{
J E A N - PAU L G A U THIER
}

D our servir le thème premier de cette revue neuve, voici une manière de com-

prendre ce qu'il en est de la liberté, du bien et du mal, à partir d'une des plus anciennes figurations de l'origine, soit celle du «Paradis Terrestre».

Un mot d'abord sur l'interprétation d'un mythe. Celui-ci, on le sait, est un récit plutôt bref qui déploie des épisodes successifs entre lesquels il crée des liens qui doivent être tenus pour nécessaires. Cependant le caractère fabuleux de ce qu'il raconte nous impose de chercher son sens ailleurs que dans ses anecdotes, mais également ailleurs qu'en une correspondance de ses éléments, considérés séparément comme des symboles, à des significations qui seraient elles-mêmes déjà données par ailleurs - comme un corpus de solutions ou une clef de second degré. La vérité du mythe n'est ni dans la conformité de son contenu manifeste à quelque réalité factuelle, ni dans l'adéquation terme à terme des éléments de ce contenu à des significations acquises et cataloguées, comme si ces dernières étaient disponibles et par avance établies. Auquel cas, le mythe ne représenterait aucun apport. La vérité du mythe ne réside ni dans la naïveté factuelle d'un acte de foi, ni dans la mise en application d'une grille de contenu, mais dans l'idée que le mythe met en œuvre et à laquelle cette œuvre donne consistance et véritable médiation; à la condition d'entendre sous ce terme d'idée non pas un thème (gagné d'avance) mais un dispositif où entrent en ligne de compte plusieurs éléments synchrones. Ainsi le mythe figure chronologiquement des instances qui s'éploient simultanément au plan de la signification; et besoin est de cette figuration mythique pour la simple raison que le dispositif évoqué est invisible, mais que c'est uniquement en un drame (temporel) qu'il peut être gravé. En ce sens, le mythe est le montage fondateur d'un mode d'être, qu'il revient à l'interprétation d'expliciter. On ne peut interpréter un mythe ni en le recevant comme une information positive ou une attestation historique, ce qui reviendrait à croire aux 
sirènes et aux fantômes, à dieu et à diable, ni en recevant ses éléments comme autant d'icônes ou de symboles autonomes, ce qui reviendrait à traiter les idées comme des choses (ainsi que le font horoscopes et tarots). Incidemment, au cours de la lecture d'un mythe, on ne doit point élire de manière univoque ce que prétend tel personnage, non plus que discréditer tel autre sous prétexte qu'il assume le rôle du coupable: ce qui est brandi à l'intérieur du mythe, sous bannière anecdotique, ne recevra pas nécessairement le traitement qu'en espèrent les personnages (ou qu'ils appréhendent). On doit au contraire repérer les implications qu'entretiennent entre elles les diverses anecdotes et les sous-entendus inhérents aux figures, si l'on veut gagner le dispositif ou la médiation qui actionne et combine divers épisodes - qui sont non moins contemporains que fatals au drame qui s'inscrit. C'est à l'interprétation qu'il revient de rassembler les diverses implications sourdement liées dans le récit, et de nous recomposer le dispositif (ou mode d'être), non apparent mais bien réel, que ce mythe essaya de graver... Tentons maintenant de dégager la matrice de cette mise en scène originelle.

Puisque le créateur impose à nos premiers parents un choix, c'est qu'il les a créés libres et qu'ils n'ont pas le choix de ne pas l'être. Ils naissent adultes au sein d'une alternative: d'un côté, le paradis sous condition, c'est-à-dire moins l'arbre de la connaissance (du bien et du mal); de l'autre, la connaissance moyennant l'expulsion du paradis, c'est-à-dire moins l'arbre de vie (éternelle).

En quoi plus précisément consiste le paradis? En la «parousie» ou la pleine présence, à commencer par le bénéfice de l'arbre de vie et donc de l'immortalité; et sur aucun autre registre on n'éprouvera quelque manque, par conséquent pas d'effort à déployer pour combler le manque. Autant dire l'absence de désir et de conquête! puisque toute production aura été rendue caduque pour ne se profiler d'emblée qu'à l'état de produit assurément disponible. Autant dire l'absence de défi et de valeur! puisqu'il n'y aura eu ni rareté, ni résistance inhérente à une mise en œuvre, ni surprise ou exception parmi des résultats préalablement fournis. Tout est par avance donné et garanti ainsi qu'à des nouveau-nés. À l'absence d'avenir (que représente l'immortalité) et à l'absence d'œuvre (que représente la plénitude) se joint l'innocence, c'est-à-dire l'absence de distinction. Car ce jardin à sécurité maximum ne se pourra conserver qu'à la condition de ne point goûter du savoir que procure l'arbre de connaissance. D'où trois questions sur ce versant paradisiaque du choix...

1. Dira-t-on qu'un interdit se voit tout de même conservé? Vivre en regard d'un interdit (ne point jouir de l'arbre de la science), n'est-ce point là réserver une place à la tentation et replonger (ou avoir toujours été plongé) dans le manque? Pas vraiment, du moins du point de vue du paradis, car la façon sous laquelle apparaît cette condition est entièrement négative: goûter de cet 
arbre entraîne la mort, leur dit le créateur. Or, on ne peut manquer d'un manque, on ne peut vouloir la mort; sous l'angle paradisiaque, la tentation semble donc ne jouir d'aucune avenue puisqu'elle équivaudrait à sa pure et simple destruction. C'est toute la contingence qui se trouve ainsi raturée par une vision qui ne la considère pas autrement qu'une maladie ou une sottise. La tradition entérinera ce point de vue en rendant le mal inconsistant, ne le considérant que comme une absence de bien - un bien par avance circonscrit que tous désireraient.

2. Et pourquoi donc Dieu profila-t-il la finitude d'un choix? Parce que seule vaut la reconnaissance d'êtres libres, qui lui auront témoigné une adhésion exclusive; une reconnaissance est exigée qui n'est autre que le dépôt de la liberté. Une donation sous condition révèle un donateur, en fait un créancier, face auquel on est d'entrée de jeu débiteur, et qui suppose une sujétion sous peine que les vivres soient coupées et les privilèges retirés. Le bénéficiaire doit être capable d'un renoncement pour que lui soit en effet accordé d'être de part en part pourvu: c'est la double signification de ce qu'implique le fait d'être pris en charge. Nul mieux que Dostoïevski n'a tiré le portrait de cette implication sous la figure de ce qu'offre mais tout à la fois exige le Grand Inquisiteur. Il offre un programme, tant matériel que spirituel, un somnifère en retour de quoi il exige une soumission intégrale. Tout sera consenti, du pain jusqu'au salut éternel via même le loisir de pécher (hormis l'orgueilleux péché de prétendre à l'autonomie), pourvu que la liberté soit remise entre les mains du grand pourvoyeur.

3. Mais pouvoir circonscrire une condition exclusive ou, autrement dit, une discrimination radicale, n'est-ce point instaurer la distinction et replonger (ou avoir toujours été plongé) dans la connaissance? Certes, et c'est ce qui atteste que nos premiers parents furent créés libres, et que même en paradis, ils avaient déjà goûté de l'arbre de la connaissance; il s'agit bien d'une projection rétroactive, condition même de la connaissance qui opère par réflexion, au futur antérieur. C'est pourquoi le paradis ne constitue qu'un versant de l'alternative; c'est pourquoi aussi il ne pourrait faire valoir son état d'innocence que via un interdit; et c'est pourquoi enfin, malgré une dépréciation de la contingence, Adam et Ève se commettront jusqu'à tâter de la mort parce qu'elle est tentante, du moins ce qu'elle implique. C'est maintenant ce qu'il y a à comprendre et qui nous mène au deuxième versant de l'alternative.

Qu'en est-il donc du fruit défendu? Il déclenche la chute dans le temps et ouvre le creuset de la mort, par où place sera faite à la reproduction des naissances. Et sur tous les autres registres, tout un chacun connaîtra le manque et l'ef- 
fort. Autant dire le désir et la conquête! puisqu'à chacun reviendra de pourvoir à ses convoitises par la mise en ouvre aléatoire de ses projets. Autant dire du défi et de la valeur! puisqu'il n'y aura pas de gain sans résistances à vaincre ni risques d'échec. Les faits et gestes étant comptés, ils seront irremplaçables. Par ailleurs le fruit de ces incertitudes et de cette précarité sera de permettre des surprises. Le régime de l'inédit va de pair avec la finitude et la discrimination.

Au niveau anecdotique on doit reconnaître l'insistance d'une dimension sexuelle attenante au geste perpétré: d'abord par l'effet d'entraînement conjugal qui mène Adam au fruit suite à la proposition d'Ève, à elle-même proposé par le serpent en érection (non encore condamné à la reptation), puis par l'effet de pudeur qui suit la consommation et qui signe que désormais la séduction devra ravir les réserves ou voler le consentement, ensuite par l'insistance que Dieu met à convaincre de douleur l'engendrement, enfin par le fait que c'est désormais le mécanisme de reproduction qui relaiera la durée d'une humanité mortelle. Bien que cet axe soit indubitablement présent dans la figuration, néanmoins sa teneur consiste plutôt en ce que, simultanément à la connaissance ou la distinction, naît ce par quoi l'homme est à l'image de Dieu, c'est-à-dire procréateur, voué au dédoublement d'une création douée d'une vie autonome. Que dit le serpent pour aiguiser la tentation et emporter les craintes? «Vous serez comme Dieu! » Est-ce un mensonge, comme pourrait le faire croire la promesse d'immortalité qui accompagne cet appât? Ce n'en est pas un au niveau immédiat, car au fil du récit, c'est Dieu qui s'empressera de leur souffler l'immortalité, l'arbre de vie demeurant accessible jusqu'à l'expulsion de l'Éden; non plus qu'au niveau médiat, puisque l'humanité fabriquera par ses œuvres un éternel recommencement. Ainsi l'humanité connaîtra-t-elle cette vertu divine d'une création continuée, soit la faculté de s'engendrer elle-même, mais à l'échelle de la contingence et sous le mode aléatoire (inhérent aux tentations) de l'incessant renouvellement.

Toujours est-il que la matrice de tout cela niche en l'arbre de la connaissance ou de la distinction du bien «et» du mal. Et c'est pour deux raisons que nous souhaitons insister sur le terme apparemment le plus anodin, soit la conjonction «et». La connaissance n'est pas définie rien qu'objectivement, par ses contenus de bien et de mal, mais par ceci qu'en son principe, elle consiste d'abord en une alternative - incessamment réitérée. De surcroît, comme l'être humain a pour caractère spécifique d'être malléable et comme la connaissance se déploie essentiellement dans le temps, voire au futur antérieur, ces domaines du bien et du mal ne sont pas complètement acquis. C'est au paradis qu'ils seraient posés par avance; mais là où, au contraire, règne la contingence, ils ne demeurent qu'atteignables et, plus préci- 
sément, à construire. Faut-il imaginer ce qui s'atteindra comme préalablement fixé, ou plutôt créé au fur et à mesure du processus d'accession. C'est a contrario de ce qui se passe au paradis qu'on peut inférer la réponse, et simultanément déterminer ce que sont le bien et le mal rendus ambivalents par l'ordre de la science.

Si en paradis les gestes sont par avance comblés au prix d'avoir cédé leur liberté, leur expérimentation et leur autonomie, c'est que toute la vie édénique se trouve d'entrée de jeu sous l'emprise et l'égide de buts - qui auront prédéterminé ces gestes jusqu'à les réduire à l'état de fonctions - , c'est que la finitude se voit ravalée par la finalité. Or, qu'est-ce qu'un tel comportement sinon celui qui déploie ses gestes de façon instrumentale! comme une pure et simple mise en application d'instances auparavant fixées par une autorité autre que soi, et qui fait de son aventure le strict déroulement d'un programme à salaire garanti. Traditionnellement on désigne un tel «moyennage» comme le fait de vendre son âme au diable, de court-circuiter l'effectuation sous le poids d'effets par ailleurs établis et réifiés. Le temps étant annulé, on comprend que s'y jouxte l'éternité. Le paradis équivaut donc, contre tout aléa, à un programme de vie «ustensilaire». Non seulement c'est au paradis que bien et mal sont par avance fixés, mais ce qui là se confond avec le bien prendra, vu hors paradis, sous l'angle du choix contingent, c'est-à-dire sous le règne alternatif de la spéculation et de la finitude, la figure du mal. Car au registre de la contingence, c'est en effet la mise en instrumentalité de l'expérience qui sera jugée aliénante, alors que sera promu le règne du hasard et du défi, soit le règne de l'innovation. Bien et mal auront donc des valeurs inverses, selon que l'on choisira ou non le paradis.

Dans le monde de la contingence, qui tout à la fois rend chaque geste irremplaçable et de ce fait responsable, on sera en revanche soumis à la mise en œuvre. L'effort de discernement en quoi consiste la distinction éthique revient à modeler les événements à coups d'hypothèses et d'expérimentations, à relancer et traduire à nouveau les acquis de l'héritage sous peine de n'être plus soi-même qu'un résultat ou un duplicatum, à répéter sans cesse ce discernement entre sujétion fonctionnelle et aventure inventive. Somme toute, le bien tel qu'ébranlé par la contingence sera, négativement dit, de contourner tout aplatissement utilitaire ou tout report utopique, et, positivement dit, de relancer la faculté d'alternative; bref, une part d'enjeu inhérente à tout défi. Autrement dit, le bien à élaborer de l'intérieur de l'alternative (le choix effectif d'Adam et Ève: $A^{2}$ dans le schème qui suit) fait cercle avec cela même qui l'a posé et rendu distinct, soit l'alternative (le loisir d'un choix: $\mathrm{A}^{1}$ dans le schème) que ce bien aura pour train de relancer! et pour laquelle relance besoin est de ce qui lui résiste, soit du mal - éteignoir de 
défi, de la répétition apathique ou de la sujétion résignée. Tel est le lot d'une situation désormais relative: nourrir l'alternative contre ce qui la nie mais la suscite. Et tel est bien ce que firent nos premiers parents, eux qui étaient déjà libres au paradis. Voilà la première raison pour laquelle nous insistions sur la conjonction «et» (entre le bien et le mal).

Résumons :

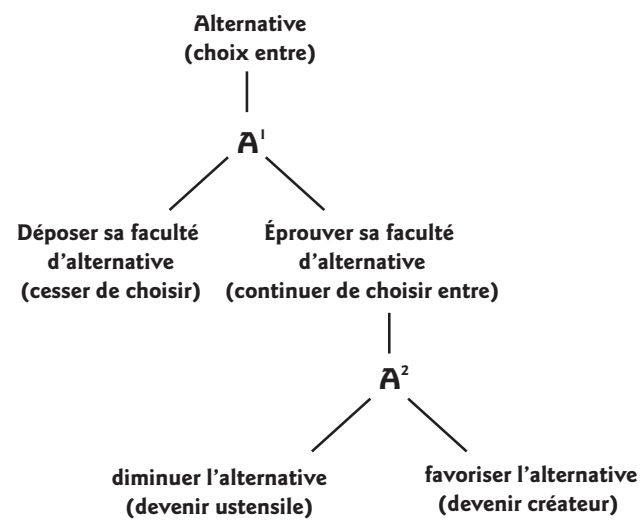

L'être humain étant radicalement sélectif, nos premiers parents furent d'entrée de jeu créés libres et obligés de choisir, ainsi le veut la cohérence du mythe. C'est pourquoi les deux alternatives, celle (générique) du mythe et celle localement inscrite en chaque geste, sont à rabattre l'une sur l'autre pour n'en former qu'une; c'est pour les besoins de la cause figurative que le mythe coule un dispositif d'origine dans les épisodes fictifs d'un commencement. S'il emprunte à l'expérience la figure chronologique du commencement, c'est en réalité pour inscrire un mode originel qui n'est point anecdotique mais qui préside à toute expérience, ce que lui permet son registre fabuleux. D'où alors cette figuration tientelle sa nécessité? Parce que là où, dans les faits et gestes nous observons une seule alternative, il y en a une deuxième tout à fait simultanée mais inobservable: le mythe nous force à déplier ce que l'expérience condense. Car celle-ci, où certes se trouvent sédimentées les deux alternatives, les condense sous la couleur factuelle en gommant leur dispositif d'origine. Le mythe montre qu'à chacune des multiples et changeantes alternatives qui nous échoient et où nous devons discriminer, une autre mais pourtant la même, spectrale pourrait-on dire, se jouxte. C'est ce que la philosophie nomme traditionnellement une idée - réputée éternelle, c'est-à-dire incessamment réitérée - , qui n'est aucunement un contenu préhensible mais en quelque sorte une insistance. À chaque liberté prise à l'égard 
d'un contenu quelconque, se joue une accentuation (ou un amoindrissement) de la liberté quant aux contenus à venir.

En n'importe quel choix, il est aisé de distinguer les objets, les sujets et les trajets d'appropriation parmi lesquels une préférence s'impose; on a même l'impression que le choix est enclenché par des consciences et qu'il est dicté par des contenus. Ce qui n'est certes pas faux, mais ce n'est pas tout ni même l'essentiel quant à la liberté. L'essentiel réside en ceci : le choix qui se fait concourt-il à une intensification ou une pétrification de la liberté, à sa relance ou à son dépôt? Car c'est en chacun des choix, aussi banal soit-il, que se renforce ou se détériore le prochain (non moins que le précédent). Voilà pourquoi tout choix, peu importe qu'il semble du côté de la morale ou non, participera toujours d'une distinction du bien et du mal, de la création ou de la stagnation de soi-même. Tout choix s'avère éthique.

Ce qui caractérise le problème, c'est qu'il est inséparable d'un choix [...] Le choix ne porte plus sur tel ou tel terme, mais sur le mode d'existence de celui qui choisit [...]. Le redoutable homme de bien ou le dévot (celui pour qui il n'y a pas à choisir), l'incertain ou l'indifférent (celui qui ne sait pas ou ne peut pas choisir), le terrible homme du mal (celui qui choisit une première fois, mais qui ne peut plus choisir ensuite, ne peut plus répéter son propre choix), enfin l'homme du choix ou de la croyance (celui qui choisit le choix ou le réitère) [...]. Cette plus haute détermination de la pensée, le choix, ce point le plus profond que tout lien avec le monde'.

Évidemment le bien et le mal seront inversés selon qu'on les considérera du point de vue du paradis, où le mal le plus aigu est identifié à l'orgueil de la connaissance et aux aléas de la contingence, ou bien du point de vue de l'humanité, où le mal coïncide avec l'utopie fonctionnelle et un programme salutiste. Si Dostoïevski a su s'acquitter de la critique du point de vue paradisiaque, c'est à Nietzsche qu'il revient d'avoir gravé une apologie de la contingence, lui qui s'interdisait d'être juge et partie, c'est-à-dire de s'élever au-dessus ou hors du monde pour le discréditer, et qui ne pouvait saluer la joie indépendamment de la souffrance ${ }^{2}$. Au carrefour de la liberté se nouent quatre exigences:

1. Gilles Deleuze, L’image-temps: Cinéma 2, Paris, Éditions de Minuit, 1985, p.230-232.

2. Pour Dostoïevski, voir Notes d'un souterrain, intro. Tzvetan Todorov, trad. Lily Denis, Paris, Éditions Aubier Montaigne, 1972 [1864], I, 8 et 9, p. 79-95, ainsi que la légende du grand inquisiteur dans Les frères Karamazov [1880]. Pour Nietzsche, voir le point de vue résolument immanent par où il énonce sa doctrine de l'éternel retour, et combien il se considère le premier penseur à rendre synonymes la finitude, le tragique et la joie (Ecce homo: comment on devient ce que l'on est, trad. Jean-Claude Hémery, Paris, Éditions Gallimard, 1974 [1888], p. 79-80). 
1. L'ardeur de la liberté suppose celle-ci pénible et ardue, car elle sera proportionnelle aux dures contraintes de l'apprentissage pour établir son savoirfaire: elle se doit d'être fidèle et de cultiver son héritage.

2. L'acquis de la liberté suppose celle-ci précaire et à conquérir, car elle sera proportionnelle aux défis et risques pour inscrire sa probation: elle se doit d'être guerrière et de se mesurer à son héritage.

3. L’originalité de la liberté suppose celle-ci inventive et originelle, car elle sera proportionnelle aux ajouts et capacités de redonner plus et autrement que reçu: elle se doit d'être créatrice et de renouveler son héritage.

4. La jouissance de la liberté suppose celle-ci tragique et surprenante, car elle sera proportionnelle aux imprévus qu'elle devra récolter: elle se doit d'être ludique et de devenir son héritage.

Quoi qu'il en soit, le jeu et l'enjeu de la liberté sont fatals, car on n'aura ni ne sera autrement fait que par ce qu'on aura dû réussir (fût-ce de convertir un échec en ressource), sans qu'un plan ne fût au départ donné - ce qui interdirait la métamorphose et garantirait l'absolution; car métamorphose et bénédiction, seule l'ouverture de la finitude les octroie. Sans la décrire si avant, voilà pourtant l'idée de liberté que comporte le mythe, au grand dam d'une tradition qui s'est échinée à comprendre ce mythe de manière aussi partiale et prétentieuse (du point de vue de Dieu) que naïve et positive (du point de vue de l'anecdote), comme s'il s'agissait de reportages.

Cette idée de liberté signe qu'en chacun des gestes, qui tous sont sélectifs, est une doublure par où ce geste, en inscrivant son élection, choisit de continuer à choisir (la contingence) ou de cesser de choisir (ce qui peut aussi signifier n'avoir point d'autres choix qu'entre des rôles prévus et achevés). À la racine et à la limite de ce dernier choix, qui se dénie, trône «le» bien, une fois pour toutes, avec sa panoplie de recettes exclusives; tandis que continuant de choisir, la liberté, incessamment relative, composera toujours avec le bien et le mal, entre le plus et le moins. Et voici la seconde raison, fondamentale, pour laquelle nous insistions tant sur la conjonction «et»: dans le monde mixte de la contingence, cette conjonction du bien «et» du mal doit être considérée de manière relative: elle signifie moins une dichotomie radicale qu'une proportion dans toute mise en œuvre ou toute mise en valeur.

Le relativisme oblige à penser d'un même tenant et en termes graduels des couples notionnels - l'âme et le corps, par exemple - , indûment séparés en substances étanches et conclus en de fieffées dichotomies. C'est d'autant plus indiqué mais difficile pour ces notions capitales et éthérées que sont vérité et fausse- 
té, bien et mal. Le relativisme, le mot le dit, oblige aussi à penser la relation comme préalable aux termes mis en relation - qu'il en aille des personnes, des moments ou des notions, si bien que ces termes par lesquels se conjugue la tension d'une amitié, d'une histoire ou d'une pensée, fussent-ils aux antipodes, doivent faire montre de leur composition, littéralement de leur entretien, en tout processus d'engendrement et de dégénérescence. Un tel point de vue est celui de la continuité. Relation, gradation et continuité sont d'un même tenant.

Ainsi du bien et du mal. Le bien passe pour être ce qu'il y a de plus intéressant: un bienfait est en effet un intérêt, c'est-à-dire un ajout, un surplus, un gain. Ce qui exige tout à la fois une vertu et une résistance... une vertu, c'est-à-dire la capacité de produire un surcroît, et une résistance par où cette vertu ou cette force témoigne de son effort - résistance qui peut cependant faire échouer cette vertu ou la tuer dans l'œuf, et qui rend le bien à chaque fois risqué. Ce méfait pourra faire en sorte que rien ne bouge, que l'inertie l'emporte ou que tout revienne du pareil à la même monotonie. Dès lors, qui ne voit pas que le méfait, c'està-dire le pas fait ou le mal fabriqué, l'empêchement de faire, le laisser défait ou la défaite invoquée pour n’avoir réussi quoi que ce soit, est tout à fait constitutif du bienfait, de la propension à faire et du parachevé? On voit donc qu'au sein de tout événement, pour peu que l'avènement soit l'enjeu, se doivent composer les occurrences d'un essor et de son avortement, de son effort et de sa défaite ou de sa victoire et de sa vanité.

Hissons-nous à un degré de plus dans la généralité. Un méfait (le mal) consiste bien sûr à empêcher qu'une création s'effectue. Certes cela se peut par des suppressions radicales, qui sont ce à quoi l'on songe en premier. On oublie souvent que ces gestes extrêmes et exceptionnels témoignent pour beaucoup d'un motif diversement déguisé et qui est celui de l'impuissance (à faire) - en lequel Nietzsche apercevait la pire des maladies ${ }^{3}$ et l'on conviendra que l'horreur de ces gestes, redoublée par leurs prétendues légitimations, tient à leur caractère programmé. Le méfait recèle des façons courantes et sournoises de s'inscrire, en ravalant par exemple ce qui est en train de se faire par le déjà tout fait. Qu'un processus de production se trouve ou se retrouve à l'état de produit fini et programmé, et c'est la réification qui l'emporte sur la réalisation et la recette sur le risque. Ce n'est pas un moindre mal, au plan d'une vie qui cesse de se faire œuvre (ou

3. Friedrich Nietzsche, La généalogie de la morale, trad. Isabelle Hildenbrand et Jean Gratien, Paris, Éditions Gallimard, 1991, I, 7; I, 14; II, 16. 
d'une société de masse qui se rêve fonctionnaliste), que la création et la joie qui lui est attenante, soit reportée au plan instrumental et noyée dans une activité de reproduction, ayant en partage la médiocrité plutôt que l'exigence, la conformité plutôt que l'aventure.

Par ailleurs, cette prédétermination du tout fait ou tout à fait fini converti en instance extérieure et prescriptive, ne la retrouve-t-on pas dans les règles et les modèles dont ne peuvent se passer les œuvres qui, pour jouer sur les lois, ne le peuvent qu'à partir de leur assise? Davantage, n'est-ce là l'épreuve obligée pour tous les enfants du monde, qui ne peuvent apprendre qu'à partir des règles, des scénarios concoctés par d'autres, qu'ils reçoivent autoritairement et préfabriqués, et qu'ils ne maîtriseront qu'à les reproduire? Tant les législateurs de tout ordre que les enfants n'auront eu le choix de commencer par se sentir serviles et dépossé74 dés, jusqu’à ce que cela leur devienne un ressort pour gagner quelque fière autonomie - à la condition de retrouver dans la sclérose autoritaire des lois une ligne de légitimité et l'aloi d'une alternative. Convertir en ressource la blessure.

Que déduire de cela? Que les divers aspects du méfait (le contrefait, le tout fait,...) relèvent d'une même affaire que les divers aspects du bienfait (refaire, parfaire,...). Parfaire est bien mais parfait l'est trop, refait l'est moins et mal fait trop peu mais refaire est mieux, etc. L'éthique ne peut être que l'éthique du bien «et» du mal, le reste n'étant affaire que de recettes morales, exclusives et univoques eu égard à ce qui n'a de cesse de renaître de ses cendres et d'ébranler les stèles. Le bien est ce qui fait non pas pareils mais mêmes le bien et le mal, et le mal ce qui fait tout autres, impératives et exclusives, une recette et une autre, encore que, d'un point de vue se voulant en deçà du bien et du mal, il ne convienne plus de concéder à l'absolu «le » bien ou «le » mal. Toujours il y aura un fin partage à tracer entre l'inouï et l'inertie.

Tout ce qui s'épanouit s'évanouit, tel est le cours unanime de la nature ou phusis, soit l'inouïe formation de la forme ou, en s'inspirant de Valéry, l'insensible croissance d'un résultat sensiblet.

4. Voir Paul Valéry, «L'homme et la coquille», Euvres, Jean Hytier (éd.), Paris, Éditions Gallimard, coll. «Bibliothèque de la Pléiade», 1957, vol.1, p. 887 et p. 902. 